Received: 30.05 .2019

Revised: 26.07.2019

Accepted: 09.08.2019

DOI: $10.17804 / 2410-9908.2019 .4 .026-037$

\title{
SPECIFIC FEATURES OF THE GRAIN STRUCTURE IN Ni-CU ALLOYS AT THE SATURATION STAGE UNDER HIGH-PRESSURE TORSION
}

\author{
A. V. Stolbovsky ${ }^{\text {a)* }}$, V. V. Popov ${ }^{\text {b) }}$, E. N. Popova ${ }^{\text {c) }}$ and S. A. Murzinova ${ }^{\text {d) }}$ \\ M. N. Miheev Institute of Metal Physics, Ural Branch of the Russian Academy of Sciences, \\ 18 S. Kovalevskoy St., Ekaterinburg, Russian Federation

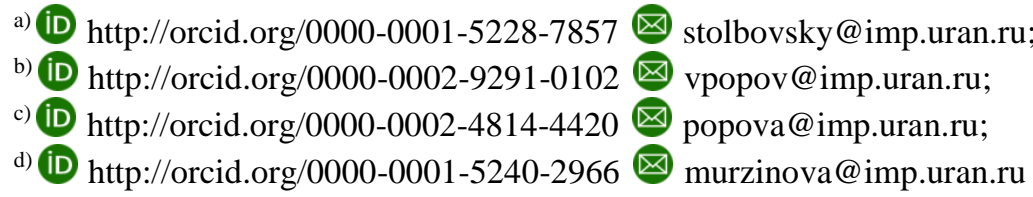 \\ *Corresponding author: E-mail: stolbovsky@imp.uran.ru \\ Address for correspondence: ul. S. Kovalevskoy, 18, 620990, Ekaterinburg, Russian Federation \\ Tel.: +7 34337835 74; fax: +7 3433745244
}

\begin{abstract}
Effect of doping in the Ni-Cu system on the structure formed under room-temperature highpressure torsion is studied, and the statistical analysis of the grain structure under steady-state deformation (saturation stage) is done. It is demonstrated that in all the alloys considered (with 10, 34 , and 90 at. $\%$ of $\mathrm{Cu}$ ) there are two groups of crystallites, in one of which a pronounced effect of relaxation processes is observed, whereas in the other one they are not revealed. The ratio of the volume fractions of these groups depends on the alloy composition and, correspondingly, on its melting temperature. It is shown that the final average grain size is formed under the effect of dominating crystallite group depending on the alloy melting temperature. The non-linearity in the crystallite sizes and volume fractions of the crystallite groups dependently on the alloy composition is observed, indicating the noticeable effect of stacking-fault energy on the structure forming under the deformation.
\end{abstract}

Keywords: copper, Ni-Cu alloys, stacking-fault energy, high-pressure torsion, submicrocrystalline structure, statistical analysis.

\section{Acknowledgment}

The work was performed within the state assignment on the theme Function, No. g/r AAAAA19-119012990095-0 and supported by the Basic Research Program of UB RAS, project No. 18-10-2-37.

\section{References}

1. Gleiter H. Nanostructured materials: basic concepts and microstructure. Acta Mater., 2000, vol. 48, no. 1, pp. 1-29. DOI: 10.1016/S1359-6454(99)00285-2.

2. Valiev R.Z., Zhilyaev A.P., Langdon T.G. Bulk nanostructured materials: Fundamentals and applications, TMS, Wiley, Hoboken, New Jersey, USA, 2013, 440 p. DOI: 10.1002/9781118742679.

3. Pippan R., Scheriau S., Hohenwarter A., Hafok M. Advantages and limitations of HPT: a review. Mater. Sci. Forum, 2008, vol. 584-586, pp. 16-21. DOI: 10.4028/www.scientific.net/MSF.584-586.16.

4. Korznikov A.V., Tyumentsev A.N., Ditenberg I.A. On the limiting minimum size of grains formed in metallic materials produced by high-pressure torsion. Phys. Met. Metallogr., 2008, vol. 106, no. 4, pp. 418-423. DOI: 10.4028/www.scientific.net/MSF.584-586.16. 
5. Pippan R., Scheriau S., Taylor A., Hafok M., Hohenwarter A., Bachmaier A. Saturation of fragmentation during severe plastic deformation. Ann. Rev. Mater. Res., 2010, vol. 40, no. 1, pp. 319-343. DOI: 10.1146/annurev-matsci-070909-104445.

6. Zhao Y.H., Liao X.Z., Zhu Y.T., Horita Z., Langdon T.G. Influence of stacking fault energy on nanostructure formation under high pressure torsion. Mater. Sci. Eng. A, 2005, vol. 410-411, pp. 188-193. DOI: 10.1016/j.msea.2005.08.074.

7. Hebesberger T., Stuwe H.P., Vorhauer A., Wetscher F., Pippan R. Structure of Cu deformed by high pressure torsion. Acta Mater., 2005, vol. 53, pp. 393-402. DOI: 10.1016/j.actamat.2004.09.043.

8. Kon'kova T.N., Mironov S.Y., Korznikov A.V. Severe cryogenic deformation of copper. Phys. Met. Metallogr., 2010, vol. 109, no. 2, pp. 171-176. DOI: 10.1134/S0031918X1002009.

9. Stolbovsky A.V., Popov V.V., Popova E.N., Pilyugin V.P. Structure, thermal stability, and state of grain boundaries of copper subjected to high-pressure torsion at cryogenic temperatures. Bulletin of the Russian Academy of Sciences: Physics, 2014, vol. 78, pp. 908-916. DOI: $10.3103 / \mathrm{S} 1062873814090299$.

10. Popov V.V., Popova E.N., Kuznetsov D.D., Stolbovskii A.V., Pilyugin V.P. Thermal Stability of Nickel Structure Obtained by High Pressure Torsion in Liquid Nitrogen. Physics of Metals and Metallography, 2014, vol. 115, pp. 682-691. DOI: 10.1134/S0031918X14070060.

11. Pilyugin V.P., Gapontseva T.M., Chashchukhina T.I., Voronova L.M., Shchinova L.I., Degtyarev M.V. Evolution of the structure and hardness of nickel upon cold and low-temperature deformation under pressure. The Physics of Metals and Metallography, 2008, vol. 105, no. 4, pp. 409-419. DOI: 10.1134/S0031918X08040157.

12. Zhang H.W., Huang X., Pippan R., Hansen N. Thermal behavior of Ni $(99.967 \%$ and $99.5 \%$ purity) deformed to an ultra-high strain by high pressure torsion. Acta Mater., 2010, vol. 58, pp. 1698-1707. DOI: 10.1016/j.actamat.2009.11.012.

13. Zhang H.W., Lu K., Pippan R., Huang X., Hansen N. Enhancement of strength and stability of nanostructured Ni by small amounts of solutes. Scripta Mater., 2011, vol. 65, pp. 481-484. DOI: 10.1016/j.scriptamat.2011.06.003.

14. Popov V.V., Stolbovsky A.V., Popova E.N., Pilyugin V.P. Structure and thermal stability of $\mathrm{Cu}$ after severe plastic deformation. Def. Diff. Forum, 2010, vol. 297-301, pp. 1312-1321. DOI: 10.4028/www.scientific.net/DDF.297-301.1312.

15. Edalati K., Fujioka T., Horita Z. Microstructure and mechanical properties of pure $\mathrm{Cu}$ processed by high-pressure torsion. Mater. Sci. Eng. A, 2008, vol. 497, pp. 168-173. DOI: 10.1016/j.msea.2008.06.039.

16. Zhao Y.H., Zhu Y.T., Liao X.Z., Horita Z., Langdon T.G. Tailoring stacking fault energy for high ductility and high strength in ultrafine grained $\mathrm{Cu}$ and its alloy. Appl. Phys. Let., 2006, vol. 89, no. 1, pp. 121906. DOI: 10.1063/1.2356310.

17. Zhu Y.T., Huang J.Y., Gubicza J., Ungar T., Wang Y.M., Ma E., Valiev R.Z. Nanostructures in Ti processed by severe plastic deformation. J. Mater. Res., 2003, vol. 18, pp. 1908-1917. DOI: 10.1557/JMR.2003.0267.

18. Dobatkin S.V., Bastarache E.N., Sakai G., Fujita T., Horita Z., Langdon T.G. Grain refinement and superplastic flow in an aluminum alloy processed by high-pressure torsion. Mater. Sci. Eng. A, 2005, vol. 408, pp. 141-146. DOI: 10.1016/j.msea.2005.07.023.

19. Qu S., An X.H., Yang H.J., Huang C.X., Yang G., Zang Q.S., Wang Z.G., Wu S.D., Zhang Z.F. Microstructural evolution and mechanical properties of $\mathrm{Cu}-\mathrm{Al}$ alloys subjected to equal channel angular pressing. Acta Mater., 2009, vol. 57, no. 5, pp. 1586-1601. DOI: 10.1016/j.actamat.2008.12.002.

20. Valiev R.Z., Gertsman V.Y., Kaibyshev O.A. Grain boundary structure and properties under external influences. Phys. Stat. Sol., 1986, vol. 97, no. 1, pp. 11-56. DOI: 10.1002/pssa.2210970102.

21. Horita Z., Smith D.J., Furukawa M., Nemoto M. An investigation of grain boundaries in submicrometer-grained Al-Mg solid solution alloys using high-resolution electron microscopy. J. Mater. Research, 1996, vol. 11, no. 8, pp. 1880-1890. DOI: 10.1557/JMR.1996.0239. 
22. Popov V.V. Mцssbauer investigations of grain-boundary diffusion and segregation. Def. Diff. Forum, 2007, vol. 263, pp. 69-74. DOI: 10.4028/www.scientific.net/DDF.258-260.497. 23. Sauvage X., Wilde G., Divinski S.V., Horita Z., Valiev R.Z. Grain boundaries in ultrafine grained materials processed by severe plastic deformation and related phenomena. Mater. Sci. Eng. A, 2012, vol. 540, pp. 1-12. DOI: 10.1016/j.msea.2012.01.080.

24. Popov V.V. Mossbauer Spectroscopy of Interfaces in Metals. Phys. Met. Metallogr., 2012, vol. 113, no. 13, pp. 1257-1289. DOI: 10.1134/S0031918X12130029.

25. Popov V.V., Sergeev A.V., Stolbovsky A.V. Emission Mussbauer spectroscopy of grain boundaries in ultrafine-grained $\mathrm{W}$ and Mo produced by severe plastic deformation. Physics of Metals and Metallography, 2017, vol. 118, pp. 354-361. DOI: 10.1134/S0031918X17040081.

26. Stolbovsky A., Farafontova E. Statistical analysis method of the grain structure of nanostructured single phase metal materials processed by high-pressure torsion. Sol. Stat. Phenomena, 2018, vol. 284, pp. 425-430. DOI: 10.4028/www.scientific.net/SSP.284.425.

27. Stolbovsky A., Farafontova E. Statistical analysis of histograms of grain size distribution in nanostructured materials processed by severe plastic deformation. Sol. Stat. Phenomena, 2018, vol. 284, pp. 431-435. DOI: 10.4028/www.scientific.net/SSP.284.431.

28. Popov V.V., Stolbovsky A.V., Popova E.N. Structure of nickel-copper alloys subjected to high-pressure torsion to saturation stage. Phys. Met. Metallogr., 2017, vol. 118, pp. 1073-1080. DOI: $10.1134 / \mathrm{S} 0031918 X 17110114$.

29. Emeis F., Peterlechner M., Divinski S.V., Wilde G. Grain boundary engineering parameters for ultrafine grained microstructures: Proof of principles by a systematic composition variation in the $\mathrm{Cu}-\mathrm{Ni}$ system. Acta Mater., 2018, vol. 50, pp. 262-272. DOI: 10.1016/j.actamat.2018.02.054.

30. Valiev R.Z., Rauch E.F., Baudelet B., Ivanishenko Yu.V. Structure and deformaton behaviour of Armco iron subjected to severe plastic deformation. Acta Mater., 1996, vol. 44, no. 12, pp. 4705-4712. DOI: 10.1016/S1359-6454(96)00156-5.

31. Horita Z., Smith D.J., Nemoto M., Valiev R.Z., Langdon T.G. Observations of grain boundary structure in submicrometer-grained $\mathrm{Cu}$ and $\mathrm{Ni}$ using high-resolution electron microscopy. J. Mater. Res., 1998, vol. 13, no. 2, pp. 446-450. DOI: 10.1557/JMR.1998.0057.

32. Zhilyaev A.P., Nurislamova G.V., Kim B.K., Baro M.D., Szpunar J.A., Langdon T.G. Experimental parameters influencing grain refinement and microstructural evolution during highpressure torsion. Acta Mater., 2003, vol. 51, pp. 753-765. DOI: 10.1016/S1359-6454(02)00466-4. Zhilyaev A.P., Ohishi K., Langdon T.G., McNelley T.R. Microstructural evolution in commercial purity aluminum during high-pressure torsion. Mater. Sci. Eng. A, 2005, vol. 410-411, pp. 277-280. DOI: 10.1016/j.msea.2005.08.044. 
Подана в журнал: 30.05 .2019

УДК 669.293:539.89:536.425

DOI: $10.17804 / 2410-9908.2019 .4 .026-037$

\title{
ОСОБЕННОСТИ ЗЕРЕННОЙ СТРУКТУРЫ НА СТАДИИ НАСЫЩЕНИЯ ПОСЛЕ КРУЧЕНИЯ ПОД ВЫСОКИМ ДАВЛЕНИЕМ В СПЛАВАХ СИСТЕМЫ НИКЕЛЬ-МЕДЬ
}

\author{
А. В. Столбовский ${ }^{\text {a** }}$, В. В. Попов ${ }^{\text {() }}$, Е. Н. Попова ${ }^{\text {в) }}$, С. А. Мурзинова ${ }^{\text {г) }}$ \\ Институт физики металлов им. М.Н. Михеева УрО РАН, \\ 18, ул. С. Ковалевской, 620108, г. Екатеринбург, Российская Федерация

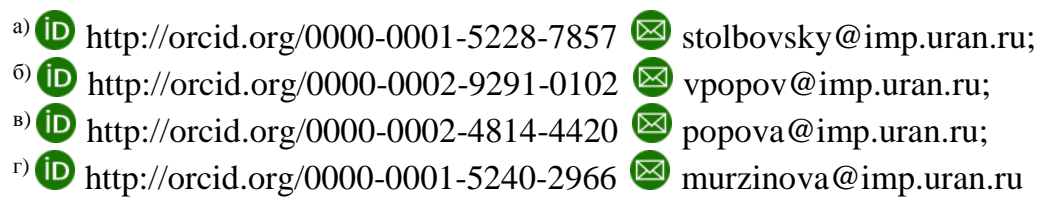 \\ *Ответственный автор. Электронная почта: stolbovsky@imp.uran.ru
}

Адрес для переписки: ул. С. Ковалевской, 18, г. Екатеринбург, 620108, Российская Федерация Тел.: +7 (343) 378-35-74; факс: (343) 374-52-44

Исследовано влияние легирования в системе $\mathrm{Ni}-\mathrm{Cu}$ на структуру при кручении под высоким давлением при комнатной температуре и произведен статистический анализ зеренной структуры в стадии установившейся деформации (насыщения). Показано, что в сплавах всех рассмотренных составов $(10,34$ и 90 ат. \% Сu) присутствуют две группы кристаллитов, в одной из которых видно значительное влияние релаксационных процессов, а в другой они не проявляются. Соотношение объемных долей этих групп зависит от состава, и от температуры плавления. Определено, что итоговый средний размер формируется под влиянием доминирующей группы кристаллитов в зависимости от температуры плавления конкретного состава. Отмечена нелинейность в изменении размеров кристаллитов и объемных долей выделенных групп в зависимости от состава сплава, что указывает на существенное влияние ЭДУ на формирующуюся при деформации структуру.

Ключевые слова: медь; сплавы $\mathrm{Ni}-\mathrm{Cu}$, энергия дефектов упаковки, кручение под высоким давлением, субмикрокристаллическая структура, статистический анализ.

\section{1. Введение}

В настоящее время объемные металлические материалы с ультрамелкозернистой (УМЗ) структурой, полученной в результате интенсивной пластической деформации (ИПД), привлекают большое внимание исследователей, поскольку они обладают уникальными прочностными, пластическими, диффузионными и другими характеристиками $[1,2]$.

Одним из наиболее эффективных и широко используемых методов ИПД является кручение под высоким давлением (КВД) [3]. В то же время достижение наноструктурного состояния, в котором преобладают кристаллиты, разделенные высокоугловыми границами, с размерами менее 100 нм, не всегда реализуемо, поскольку наступает стадия насыщения, при которой рост степени деформации уже не приводит ни к дальнейшему измельчению структуры, ни к упрочнению материала $[4,5]$. На стадии насыщения наступает равновесие между процессами генерации и аннигиляции дефектов кристаллической решетки. Ключевым процессом, ограничивающим измельчение кристаллической структуры, является миграция границ зерен [5], а наименьшие размеры зерен, достижимые при КВД, определяются прежде всего самим материалом - его кристаллической решеткой, температурой плавления и энергией дефектов упаковки (ЭДУ) [4, 6]. 
В многочисленных публикациях об эволюции структуры меди и никеля при КВД [7-15] представлено, что эти металлы способны деформироваться без разрушения даже при очень больших степенях деформации, в том числе в условиях низких температур. При этом авторы отмечают значительное влияние температуры на возможность формирования нанокристаллической структуры [9-11]. Другим фактором помимо температуры деформирования, оказывающим существенное влияние на результирующую структуру при ИПД, является энергия дефектов упаковки (ЭДУ) [6, 16]. При высоких значениях ЭДУ доминирующую роль при формировании структуры играют дислокации и их подвижность [17, 18]. В материалах с низкой ЭДУ преобладает двойникование, и меньший размер зерна в одинаковых условиях ИПД наблюдается при более низкой ЭДУ $[6,16,19]$.

Наряду с ультрамелкозернистой структурой еще одним важнейшим фактором, влияющим на результирующие свойства материалов, полученных ИПД, является присутствие в них так называемых «неравновесных» границ зерен, которые характеризуются избыточной энергией, повышенным свободным объемом и следовательно, дальнодействующими полями напряжений [20]. В ряде работ подтверждается, что состояние «неравновесных» (деформационномодифицированных) границ зерен после ИПД отличается от состояния границ рекристаллизационного происхождения в крупнозернистых материалах [21-25].

В недавних работах [26, 27] предложены новые подходы на основе аппарата математической статистики для анализа зеренной структуры в материалах, полученных ИПД, где показано, что наблюдаемые распределения могут содержать несколько наборов зерен с различными характеристиками. Это позволяет проводить глубокий анализ поведения выделенных групп кристаллитов при изменении условий наблюдений, например при нагреве или изменении химического состава.

Цель настоящей работы - расширить имеющиеся представления [28, 29] о сплавах системы $\mathrm{Cu}-\mathrm{Ni}$ на основе анализа структуры, сформировавшейся на стадии насыщения.

\section{2. Материал и методика исследования}

Для исследований использовались сплавы системы $\mathrm{Ni}-\mathrm{Cu}$ трех составов $(10,34$ и 90 ат. \% Сu), а также поликристаллический никель чистотой 99,6 \% и поликристаллическая медь марки М1, подвергнутые интенсивной пластической деформации. Исходные образцы диаметром 10 мм и толщиной 0,5 мм деформировались на 5 оборотов методом кручения под высоким давлением в наковальнях Бриджмена при комнатной температуре. Среднее давление осевого сжатия дисковых образцов составляло 6 ГПа, что надежно исключало проскальзывание при кручении; угловая скорость вращения наковален 0,3 об./мин. Предварительные исследования показали, что при деформации на 5 об. для всех исследуемых сплавов достигается стадия насыщения.

Структуру деформированных образцов изучали в просвечивающих электронных микроскопах JEM-200CX и Philips CM30 с последующей обработкой снимков с помощью программного комплекса SIAMS-600. При этом строились гистограммы распределения кристаллитов по размерам. Число кристаллитов, по которым строились гистограммы, было не менее 250, и погрешность определения среднего размера кристаллитов составляет при этом доли процента.

\section{3. Результаты исследования и их обсуждение}

Формирование структуры при ИПД, в том числе при использовании метода КВД, согласно многочисленным исследованиям [30-33], проходит по следующему механизму: с увеличением величины деформации происходит накопление дислокаций и их локализация в стенках ячеек с дальнейшим увеличением углов разориентации, что в дальнейшем приводит к образованию УМЗ-структуры. Дальнейшая деформация ведет к активации ротационных мод, что обеспечивает наступление стадии насыщения, когда дальнейшее возрастание 
величины деформации уже не приводит к существенным изменениям структуры. При этом определяющим фактором для фрагментации структуры является подвижность дислокаций, которая зависит от температуры деформации и ЭДУ. Для понижения температуры используют деформирование в жидком азоте $[10,11,14]$, а для изменения ЭДУ, как правило, прибегают к легированию [28, 29].

Ранее в работе [28] было рассмотрено формирование структуры на стадии насыщения в системе $\mathrm{Cu}-\mathrm{Ni}$ в общем и не затрагивался детальный анализ формирующихся структур. В то же время в работах [26, 27] предложены подходы, позволяющие выделять отдельные группы кристаллитов, обобщенные определенными признаками, такими как средний размер зерна и величина разброса значений относительно его среднего значения. Было показано, что материалы, наноструктурированные методом КВД, в результирующей структуре могут иметь несколько групп кристаллитов с отличным друг от друга поведением при нагреве. Следовательно, можно говорить о различном состоянии границ зерен для данных групп кристаллитов, поскольку именно их подвижность и определяет характер поведения кристаллитов при дальнейшем нагреве. Кроме того, в работе [3] показано, что при ИПД методом КВД на стадии насыщения возможна реализация механизма релаксации, заключающегося в перестройке зеренной структуры путем движения уже сформированных межкристаллитных границ непосредственно в процессе деформации.

На рис. 1 представлены гистограммы распределения зерен по размерам для сплавов системы Ni-Cu с разложением на отдельные группы кристаллитов.

Разделение по группам производилось на основе подхода, представленного в работе [27]. Группы кристаллитов аппроксимировались моделью, представленной в работе [26], следующего вида:

$$
f(x)=A \cdot \frac{1}{x} \cdot \frac{1}{\sqrt{2 \pi} \cdot \sigma} \cdot e^{-\frac{(\ln x-\mu)^{2}}{2 \sigma^{2}}}+B \cdot \frac{1}{\sqrt{2 \pi} \cdot S} \cdot e^{-\frac{(x-M)^{2}}{2 S^{2}}}
$$

где $M=e^{\mu+\frac{\sigma^{2}}{2}}$ - среднее значение для компоненты модели нормального распределения, рассчитанное из соответствующей величины для логарифмического нормального распределения; $S$ - стандартное отклонение, также рассчитанное из соответствующей величины для логарифмического нормального распределения; $\mu$ и $\sigma$ - соответственно среднее значение и стандартное отклонение для логнормального распределения; $A$ и $B$ - размерные факторы, определяющие вклад соответствующей компоненты.

Результаты расчетов представлены на рис. 2. Обобщение выделенных распределений кристаллитов с отнесением к той или иной группе производилось из принципа плавного изменения всех определяемых характеристик в совокупности.

При анализе полученных результатов необходимо учесть два важных фактора, влияющих на результирующую структуру исследуемых составов. Так, при легировании никеля медью можно ожидать образование менее дисперсной структуры, поскольку гомологическая температура такого сплава повышается за счет значительно более низкой температуры плавления легирующего элемента. В то же время с увеличением содержания меди ЭДУ сплава понижается, поскольку у меди ЭДУ почти в два раза ниже, чем у никеля, что должно влиять в противоположном направлении в сравнении с влиянием гомологической температуры. Таким образом, результирующая структура будет зависеть от совокупности влияния обоих факторов.

По результатам разложения можно выделить две отдельные группы кристаллитов, которые можно охарактеризовать не только разной объемной долей и средним размером для разных составов, но и характером их изменения в зависимости от состава. 


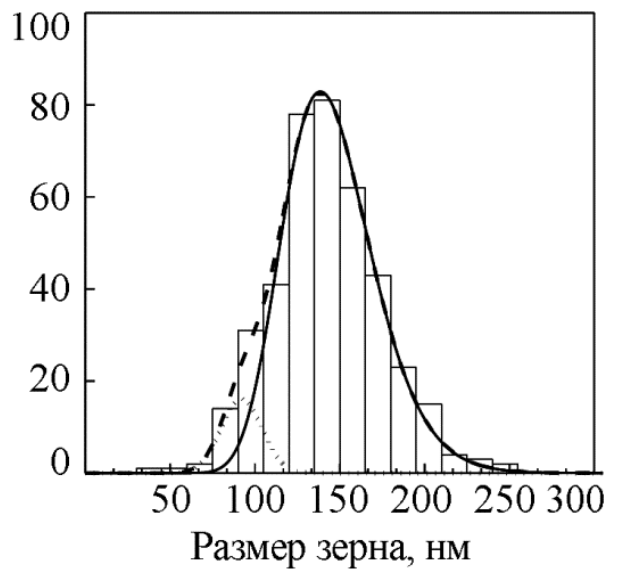

$a$

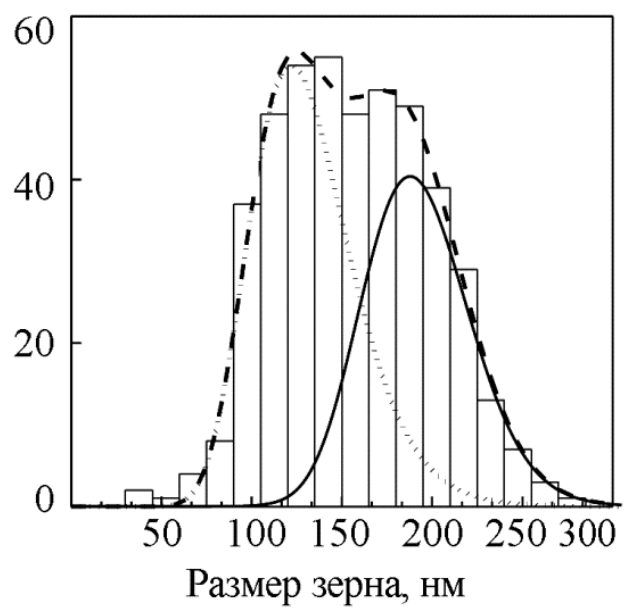

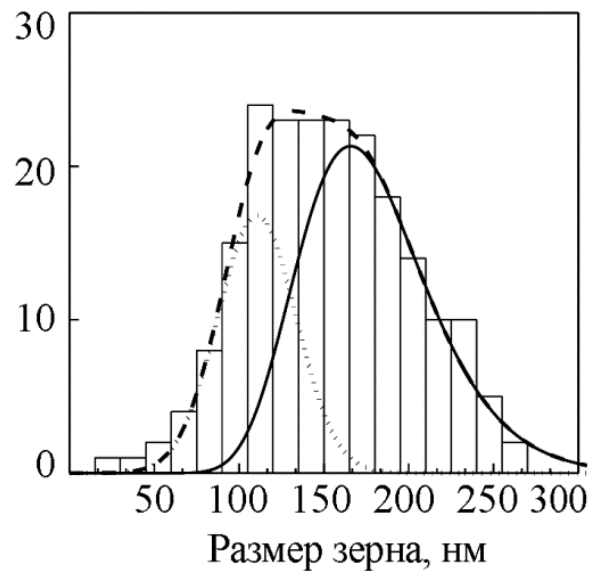

6

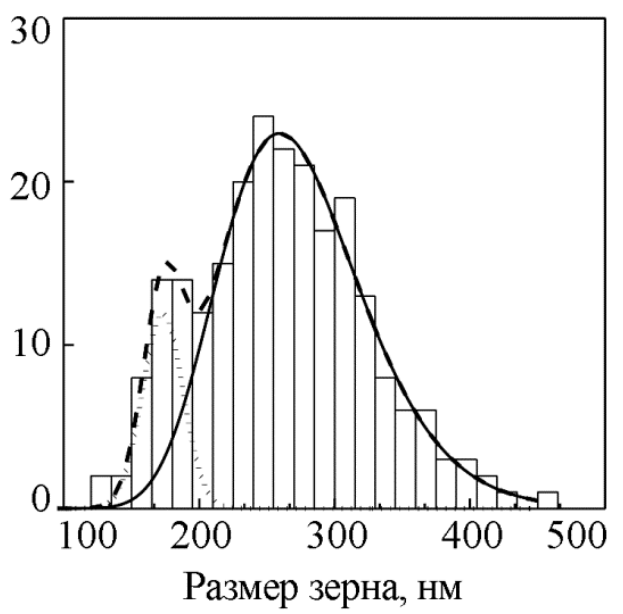

B 2

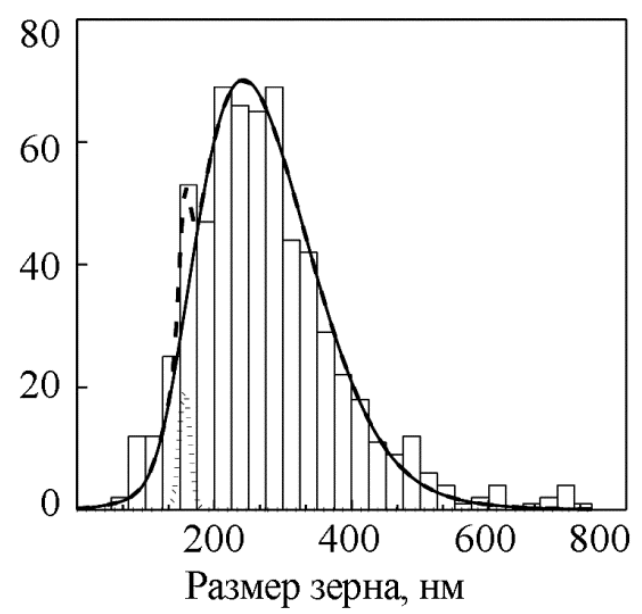

\begin{tabular}{|c|}
\hline$--\cdot 1+2$ \\
\hline$\cdots . .$. \\
\hline \\
\hline \\
\hline
\end{tabular}

$\partial$

Рис. 1. Гистограммы распределения зерен по размерам для различных составов системы $\mathrm{Cu}-\mathrm{Ni}$ после КВД на 5 оборотов при комнатной температуре с выделением отдельных групп зерен: $a-\mathrm{Ni} ; \sigma-\mathrm{Ni}-10 \mathrm{Cu} ; \varepsilon-\mathrm{Ni}-34 \mathrm{Cu} ; 2-\mathrm{Cu}-10 \mathrm{Ni} ; \partial-\mathrm{Cu}$ 


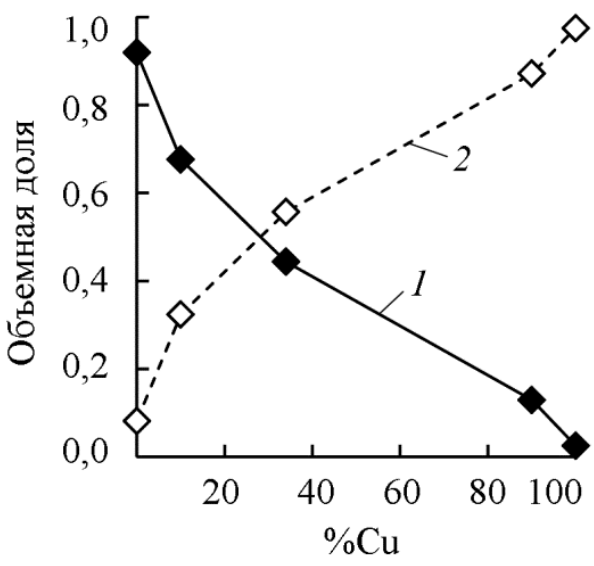

$a$

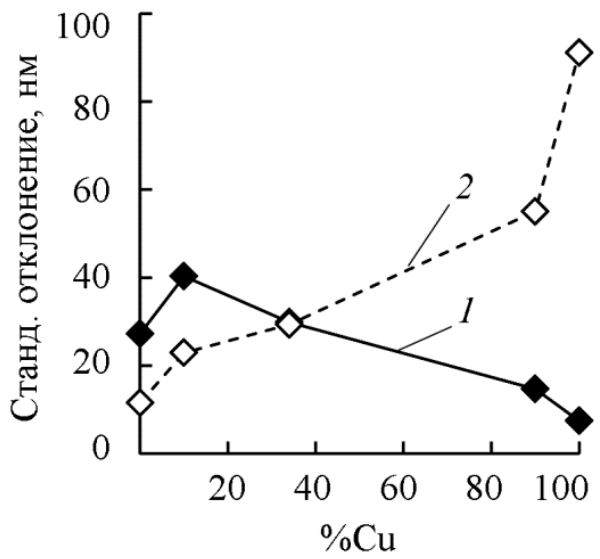

B

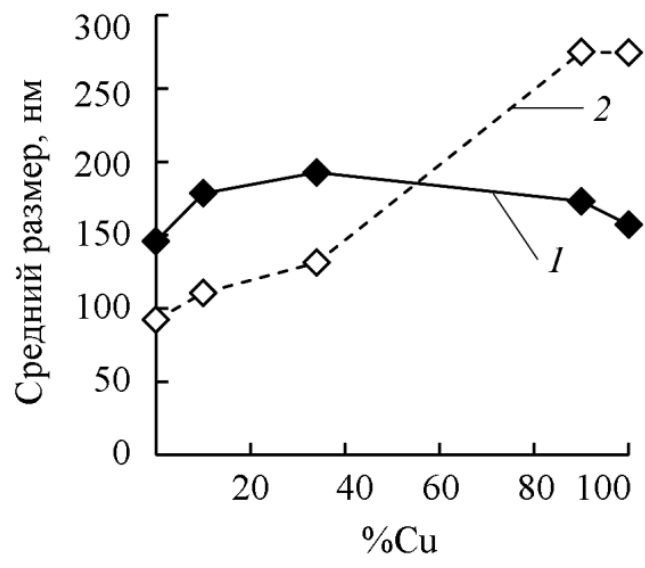

$\sigma$

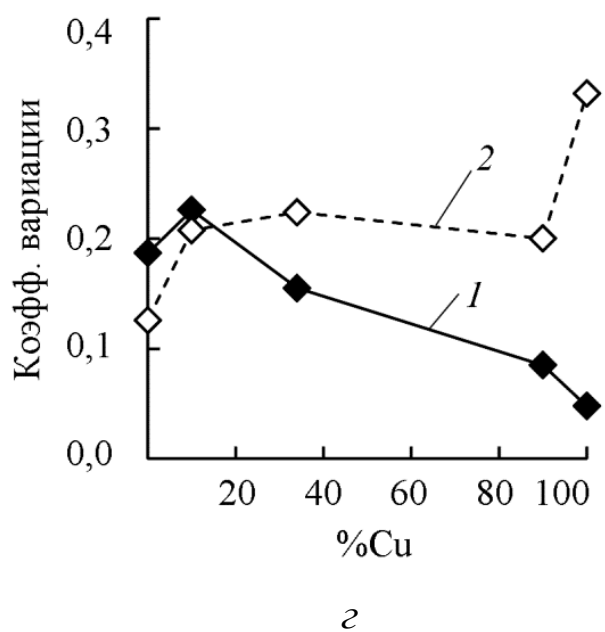

Рис. 2. Результаты расчетов величин модели, полученных из обработки гистограмм распределения зерен по размерам для сплавов различного состава системы $\mathrm{Ni}-\mathrm{Cu}$ : где 1 и 2 - группы кристаллитов

Если проследить изменения объемных долей групп от состава (рис. 2), то видно, что для крайней левой точки, отвечающей чистому $\mathrm{Ni}$, отмечается доминирование группы 1 со средним размером кристаллитов 145 нм и небольшая доля группы 2 с размером кристаллитов около 90 нм. Для другой крайней точки, отвечающей чистой Сu, уже фиксируется доминирование группы 2 , в то же время группы меняются местами по положению на графике зависимости среднего размера кристаллитов от состава. Средний размер кристаллитов группы 1 меняется незначительно и в крайней правой точке составляет 157 нм, в то время как для группы 2 средний размер значительно возрастает и составляет 274 нм.

Это можно объяснить, если взять в рассмотрение склонность сплавов разного состава к прохождению релаксационных процессов. Так, для чистого $\mathrm{Ni}$, имеющего значительно большую температуру плавления, проявление релаксационных процессов как во время деформации, так и после нее, как правило, не наблюдается [10-13]. В то же время для чистой $\mathrm{Cu}$ наблюдается существенное влияние релаксационных процессов на результирующую структуру [14]. В результате доминирующей для чистого Ni будет группа кристаллитов, не претерпевших релаксационных процессов, в то время как для чистой $\mathrm{Cu}$ доминирующей является группа кристаллитов, сформированных под влиянием релаксационных процессов, что согласуется с данными, полученными в работах [7, 14, 15 и др.]. Следовательно, выделенную группу 1 можно охарактеризовать как совокупность кристаллитов, не претерпевших релак- 
сационные процессы, а группу 2 - как кристаллиты, сформированные под существенным влиянием релаксационных процессов. При этом объемные доли этих групп изменяются в зависимости от состава и температуры плавления, что можно наблюдать на полученных зависимостях (рис. 2).

Другую особенность, указывающую на различие выделенных групп кристаллитов, можно наблюдать на зависимостях среднего размера зерна. Для группы 2 характерно планомерное увеличение среднего размера кристаллитов с ростом содержания $\mathrm{Cu}, \mathrm{u}$, соответственно, снижения температуры плавления, в то время как для группы 1 , сформированной кристаллитами без существенного влияния релаксационных процессов, отмечается незначительное изменение среднего размера вне зависимости от исследуемого состава.

Также необходимо отметить нелинейность в изменении размера кристаллитов и объемных долей выделенных групп кристаллитов в зависимости от состава. Как отмечалось ранее [28], если бы на результирующие параметры структуры влиял только один параметр, например гомологическая температура, то средний размер и микротвердость изменялись бы в зависимости от состава линейно (по правилу смесей). Тот факт, что этого не происходит, свидетельствует о том, что на структуру влияет не только температура, но и ЭДУ, изменение которой в зависимости от состава может не подчиняться правилу смесей и быть нелинейным [29].

\section{4. Заключение}

Исследовано влияние легирования в системе $\mathrm{Ni}-\mathrm{Cu}$ на структуру, формирующуюся при кручении под высоким давлением в условиях комнатной температуры, и произведен статистический анализ зеренной структуры в стадии установившейся деформации (насыщения).

Показано, что во всех рассмотренных составах присутствуют две группы кристаллитов, одна из которых сформирована из кристаллитов при значительном влиянии релаксационных процессов, а другая - зернами без значительного влияния релаксационных процессов. Соотношение объемных долей этих групп зависит от состава и, следовательно, от температуры плавления.

Установлено, что итоговый средний размер формируется под влиянием доминирующей группы кристаллитов в зависимости от температуры плавления конкретного состава. Чем ниже температура плавления, тем больше объемная доля, сформированная кристаллитами при значительном влиянии релаксационных процессов, и тем больше итоговый средний размер, рассчитанный по всем кристаллитам.

Отмечена нелинейность в изменении как размера кристаллитов, так и объемных долей выделенных групп кристаллитов в зависимости от состава сплава. Это указывает не только на влияние температуры плавления, но и существенное влияние ЭДУ, изменение которой в зависимости от состава может не подчиняться правилу смесей и быть нелинейным, что следует учитывать при создании сплавов с заданным комплексом свойств.

\section{Благодарность}

Работа выполнена в рамках государственного задания по теме «Функция» № г/p АAAA-A19-119012990095-0 при поддержке программы фундаментальных исследований УрО РАН (проект № 18-10-2-37). 


\section{Литература}

1. Gleiter H. Nanostructured materials: basic concepts and microstructure // Acta Mater. 2000. - Vol. 48, no. 1. - P. 1-29. - DOI: 10.1016/S1359-6454(99)00285-2.

2. Valiev R. Z., Zhilyaev A. P., Langdon T. G. Bulk nanostructured materials: Fundamentals and applications. - TMS, Wiley : Hoboken, New Jersey, USA, 2013. - P. 440. DOI: $10.1002 / 9781118742679$.

3. Advantages and limitations of HPT: a review / R. Pippan, S. Scheriau, A. Hohenwarter, M. Hafok // Mater. Sci. Forum. - 2008. - Vol. 584-586. - P. 16-21. - DOI: 10.4028/www.scientific.net/ /MSF.584-586.16.

4. Korznikov A. V., Tyumentsev A. N., Ditenberg I. A. On the limiting minimum size of grains formed in metallic materials produced by high-pressure torsion // Phys. Met. Metallogr. 2008. - Vol. 106, no. 4. - P. 418-423. - DOI: 10.4028/www.scientific.net/MSF.584-586.16.

5. Saturation of fragmentation during severe plastic deformation / R. Pippan, S. Scheriau, A. Taylor, M. Hafok, A. Hohenwarter, A. Bachmaier // Ann. Rev. Mater. Res. - 2010. - Vol. 40, no. 1. - P. 319-343. - DOI: 10.1146/annurev-matsci-070909-104445.

6. Influence of stacking fault energy on nanostructure formation under high pressure torsion / Y. H. Zhao, X. Z. Liao, Y. T. Zhu, Z. Horita, T. G. Langdon // Mater. Sci. Eng. A. - 2005. Vol. 410-411. - P.188-193. - DOI: 10.1016/j.msea.2005.08.074.

7. Structure of $\mathrm{Cu}$ deformed by high pressure torsion / T. Hebesberger, H. P. Stuwe, A. Vorhauer, F. Wetscher, R. Pippan // Acta Mater. - 2005. - Vol. 53. - P. 393-402. DOI: $10.1016 /$ j.actamat.2004.09.043.

8. Kon'kova T. N., Mironov S. Y., Korznikov A. V. Severe cryogenic deformation of copper // Phys. Met. Metallogr. - 2010. - Vol. 109, no. 2. - P. 171-176. - DOI: 10.1134/S0031918X1002009.

9. Structure, thermal stability, and state of grain boundaries of copper subjected to highpressure torsion at cryogenic temperatures / A. V. Stolbovsky, V. V. Popov, E. N. Popova, V. P. Pilyugin // Bulletin of the Russian Academy of Sciences: Physics. - 2014. - Vol. 78. P. 908-916. - DOI: 10.3103/S1062873814090299.

10. Thermal Stability of Nickel Structure Obtained by High Pressure Torsion in Liquid Nitrogen / V. V. Popov, E. N. Popova, D. D. Kuznetsov, A. V. Stolbovskii, V. P. Pilyugin // Physics of Metals and Metallography. - 2014. - Vol. 115. - P. 682-691. - DOI: 10.1134/S0031918X14070060.

11. Evolution of the structure and hardness of nickel upon cold and low-temperature deformation under pressure / V. P. Pilyugin, T. M. Gapontseva, T. I. Chashchukhina, L. M. Voronova, L. I. Shchinova, M. V. Degtyarev // The Physics of Metals and Metallography. - 2008. - Vol. 105, no. 4. - P. 409-419. - DOI: 10.1134/S0031918X08040157.

12. Thermal behavior of $\mathrm{Ni}(99.967 \%$ and $99.5 \%$ purity) deformed to an ultra-high strain by high pressure torsion / H. W. Zhang, X. Huang, R. Pippan, N. Hansen // Acta Mater. - 2010. Vol. 58. - P. 1698-1707. - DOI: 10.1016/j.actamat.2009.11.012.

13. Enhancement of strength and stability of nanostructured Ni by small amounts of solutes / H. W. Zhang, K. Lu, R. Pippan, X. Huang, N. Hansen // Scripta Mater. - 2011. - Vol. 65. P. 481-484. - DOI: 10.1016/j.scriptamat.2011.06.003.

14. Structure and thermal stability of $\mathrm{Cu}$ after severe plastic deformation / V. V. Popov, A. V. Stolbovsky, E. N. Popova, V. P. Pilyugin // Def. Diff. Forum. - 2010. - Vol. 297-301. P. 1312-1321. - DOI: 10.4028/www.scientific.net/DDF.297-301.1312. 
15. Edalati K., Fujioka T., Horita Z. Microstructure and mechanical properties of pure $\mathrm{Cu}$ processed by high-pressure torsion // Mater. Sci. Eng. A. - 2008. - Vol. 497. - P. 168-173. DOI: $10.1016 /$ j.msea.2008.06.039.

16. Tailoring stacking fault energy for high ductility and high strength in ultrafine grained $\mathrm{Cu}$ and its alloy / Y. H. Zhao, Y. T. Zhu, X. Z. Liao, Z. Horita, T. G. Langdon // Appl. Phys. Let. 2006. - Vol. 89, no. 12. - P. 121906. - DOI: 10.1063/1.2356310.

17. Nanostructures in Ti processed by severe plastic deformation / Y. T. Zhu, J. Y. Huang, J. Gubicza, T. Ungar, Y. M. Wang, E. Ma, R. Z. Valiev // J. Mater. Res. - 2003. - Vol. 18. P. 1908-1917. - DOI: 10.1557/JMR.2003.0267.

18. Grain refinement and superplastic flow in an aluminum alloy processed by high-pressure torsion / S. V. Dobatkin, E. N. Bastarache, G. Sakai, T. Fujita, Z. Horita, T. G. Langdon // Mater. Sci. Eng. A. - 2005. - Vol. 408. - P. 141-146. - DOI: 10.1016/j.msea.2005.07.023.

19. Microstructural evolution and mechanical properties of $\mathrm{Cu}-\mathrm{Al}$ alloys subjected to equal channel angular pressing / S. Qu, X. H. An, H. J. Yang, C. X. Huang, G. Yang, Q. S. Zang, Z. G. Wang, S. D. Wu, Z. F. Zhang // Acta Mater. - 2009. - Vol. 57, no. 5. - P. 1586-1601. DOI: 10.1016/j.actamat.2008.12.002.

20. Valiev R. Z., Gertsman V. Y., Kaibyshev O. A. Grain boundary structure and properties under external influences // Phys. Stat. Sol. - 1986. - Vol. 97, no. 1. - P. 11-56. DOI: $10.1002 /$ pssa.2210970102.

21. An investigation of grain boundaries in submicrometer-grained $\mathrm{Al}-\mathrm{Mg}$ solid solution alloys using high-resolution electron microscopy / Z. Horita, D. J. Smith, M. Furukawa, M. Nemoto // J. Mater. Research. - 1996. - Vol. 11, no. 8. - P. 1880-1890. - DOI: 10.1557/JMR.1996.0239.

22. Popov V. V. Mцssbauer investigations of grain-boundary diffusion and segregation // Def. Diff. Forum. - 2007. - Vol. 263. - P. 69-74. - DOI: 10.4028/www.scientific.net/DDF.258260.497.

23. Grain boundaries in ultrafine grained materials processed by severe plastic deformation and related phenomena / X. Sauvage, G. Wilde, S. V. Divinski, Z. Horita, R. Z. Valiev // Mater. Sci. Eng. A. - 2012. - Vol. 540. - P. 1-12. - DOI: 10.1016/j.msea.2012.01.080.

24. Popov V. V. Mossbauer Spectroscopy of Interfaces in Metals // Phys. Met. Metallogr. 2012. - Vol. 113, no. 13. - P. 1257-1289. - DOI: 10.1134/S0031918X12130029.

25. Popov V. V., Sergeev A. V., Stolbovsky A. V. Emission Mцssbauer spectroscopy of grain boundaries in ultrafine-grained $\mathrm{W}$ and Mo produced by severe plastic deformation // Physics of Metals and Metallography. - 2017. - Vol. 118. - P. 354-361. - DOI: 10.1134/S0031918X17040081.

26. Stolbovsky A., Farafontova E. Statistical analysis method of the grain structure of nanostructured single phase metal materials processed by high-pressure torsion // Sol. Stat. Phenomena. - 2018. - Vol. 284. - P. 425-430. - DOI: 10.4028/www.scientific.net/SSP.284.425.

27. Stolbovsky A., Farafontova E. Statistical analysis of histograms of grain size distribution in nanostructured materials processed by severe plastic deformation // Sol. Stat. Phenomena. - 2018. Vol. 284. - P. 431-435. - DOI: 10.4028/www.scientific.net/SSP.284.431.

28. Popov V. V., Stolbovsky A. V., Popova E. N. Structure of nickel-copper alloys subjected to high-pressure torsion to saturation stage // Phys. Met. Metallogr. - 2017. - Vol. 118. - P. 10731080. - DOI: 10.1134/S0031918X17110114.

29. Grain boundary engineering parameters for ultrafine grained microstructures: Proof of principles by a systematic composition variation in the $\mathrm{Cu}-\mathrm{Ni}$ system / $\mathrm{F}$. Emeis, M. Peterlechner, S. V. Divinski, G. Wilde. // Acta Mater. - 2018. - Vol. 150. - P. 262-272. DOI: 10.1016/j.actamat.2018.02.054. 
30. Structure and deformaton behaviour of Armco iron subjected to severe plastic deformation / R. Z. Valiev, E. F. Rauch, B. Baudelet, Yu. V. Ivanishenko // Acta Mater. - 1996. - Vol. 44, no. 12. - P. 4705-4712. - DOI: 10.1016/S1359-6454(96)00156-5.

31. Observations of grain boundary structure in submicrometer-grained $\mathrm{Cu}$ and $\mathrm{Ni}$ using highresolution electron microscopy / Z. Horita, D. J. Smith, M. Nemoto, R. Z. Valiev, T. G. Langdon // J. Mater. Res. - 1998. - Vol. 13, no. 2. - P. 446-450. - DOI: 10.1557/JMR.1998.0057.

32. Experimental parameters influencing grain refinement and microstructural evolution during high-pressure torsion / A. P. Zhilyaev, G. V. Nurislamova, B. K. Kim, M. D. Baro, J. A. Szpunar, T. G. Langdon // Acta Mater. - 2003. - Vol. 51. - P. 753-765. - DOI: 10.1016/S13596454(02)00466-4.

33. Microstructural evolution in commercial purity aluminum during high-pressure torsion / A. P. Zhilyaev, K. Ohishi, T. G. Langdon, T. R. McNelley // Mater. Sci. Eng. A. - 2005. Vol. 410-411. - P. 277-280. - DOI: 10.1016/j.msea.2005.08.044. 